\title{
Individualistic and Collectivistic Values, Age and Length of Residency Among Chinese Australian Immigrants
}

\author{
Tan Kan $\mathrm{Ku}^{1}$, Michael $\mathrm{Ha}^{2}$, * \\ ${ }^{1}$ Department of Nursing \& Paramedicine, Victoria Polytechnic, Melbourne, Victoria, Australia \\ ${ }^{2}$ Department of Financial Engineering, Sino-US College, Beijing Institute of Technology, Zhuhai, China
}

Email address:

Michael.Ha@suc.bitzh.edu.cn (M. Ha), maha2668@yahoo.com (M. Ha)

${ }^{*}$ Corresponding author

\section{To cite this article:}

Tan Kan Ku, Michael Ha. Individualistic and Collectivistic Values, Age and Length of Residency Among Chinese Australian Immigrants. Psychology and Behavioral Sciences. Vol. 6, No. 5, 2017, pp. 90-95. doi: 10.11648/j.pbs.20170605.13

Received: June 1, 2017; Accepted: June 23, 2017; Published: October 18, 2017

\begin{abstract}
It had been documented in the literature that Chinese people endorsed more highly collectivist values when compared with their Western counterparts who endorsed more individualistic values (bi-cultural differences). The aim of the study was to examine if the same pattern existed among three groups of Chinese people (mono culture) in Australia, without comparing them with another ethnic group. A method using a 26-item survey labelled as Cultural Value Scale (CVS) was administered to 138 Chinese Australians. Principal component analyses (with varimax rotation) were used to identify underlying dimensionality in the correlations of items. Scales were constructed from the final solution and Cronbach's alpha calculated. Subscale score variations were analysed to examine the discriminant validity of the subscales. Our results using Principal Component Analysis revealed four dimensions accounting for 47 percent of the variation within items. Four factors were derived. These were labelled Collectivist Family Conformity (CFC), Collectivist Family Interdependence (CFI), Individualist Self Assertion (ISA) and Individualist Self Opinion (ISO). Developed as subscales, reliability analysis indicated moderately high internal consistency with respective alpha coefficients of $0.77,0.67,0.54$, and 0.61 . We conclude that Chinese Australians endorsed more collectivist values than individualist values, correlated positively with the literature.
\end{abstract}

Keywords: Chinese Australian, Cultural Values, Collectivistic, Individualistic

\section{Introduction}

Cross-cultural psychologists and social researchers have identified that the Individualism-Collectivism Constructs was the commonly used measure for comparing cross-national values among different cultural groups [1] [2] [3]. Countries which are most individualistic are USA, Canada, United Kingdom, Australia and New Zealand, whereas Asian, European and African nations are more collectivistic in their values system. Asian subjects refer to Japanese, Taiwanese, Filipino, Vietnamese, Thais, as well as Chinese (born outside mainland China), the Singaporean, Hong Kong people and Malaysian who had been born under the British colonial influence. These latter groups of Asians, though having received western education could have maintained Chinese values, which are collectivistic in nature, partially influenced by Confucianism, a complex set of political, moral, social and philosophical system that had immerse influence on the history and culture of China over 2000 years [4] [5] [6]. The literature have identified that Chinese endorsed more highly collectivist values when compared with western people at a national and international levels over a wide spectrum of research settings [7 ] [8] [9]. During the design of a larger $\mathrm{PhD}$ study of examining the stigma of mental illness, based on a conceptual model incorporating cultural values, social comparison theory, saving face in Chinese Australian community, the CVS was administered to 138 ethnic Chinese. This paper reports the outcome of using the CVS in assessing cultural values within Chinese Australian community.

\section{Methods}

\subsection{Subjects}

One hundred and thirty-eight Chinese professionals 
participated in this study. Four subjects $(2.9 \%)$ were born locally in Australia whilst 134 subjects (97.1\%) were immigrants. Participants who were health workers (doctors, nurses and social workers) accounted for $40.6 \%$, whilst $34.1 \%$ engaged in commercial, legal, information technology and engineering, and $21.6 \%$ were in teaching and learning sectors including teachers, tertiary students, researcher and translator. Ninety-six (68.6\%) were females compared with 42 males (31.4\%).

Age range was from 18 to 65 . The mean age was 39.3.
Due to the diverse age range of participants $(n=138)$ in the sample, age was specified into three categories from the youngest to the oldest: Age Group 1, AGE 1 (18-34), Age Group 2, AGE 2 (35-49) and Age Group 3, AGE 3 (50+). Exact p-values for the Chi-Square test of association were examined using Monte Carlo methods when the assumption of less than $20 \%$ of cells having expected counts below five was violated. Otherwise, Chi-square asymptotic p-values were reported. Table 1 shows the sample characteristics.

Table 1. Characteristics of the Sample $(N=138)$ \#.

\begin{tabular}{|c|c|c|c|c|}
\hline & DECOA 1 (1950-1979) $n=25$ & DECOA 21 (1980-1999) n=53 & DECOA $3(2000-2009) n=56$ & $\chi^{2} /$ F value \\
\hline Mean Age (SD) & $55.3(9.4)$ & $42.2(13.4)$ & $30.6(10.4)$ & $\mathrm{F}(2,131)=41.2 * * *$ \\
\hline Sex (male/female) & $10 / 15$ & $14 / 39$ & $18 / 38$ & $1.5 \mathrm{~ns}$ \\
\hline Education Level (\%) & & & & $22.7 * * 1$ \\
\hline Secondary & 8.0 & 0 & 0 & \\
\hline Completed secondary & 8.0 & 11.3 & 25.0 & \\
\hline Completed trade & 12.0 & 9.4 & 5.4 & \\
\hline Postgraduate & 16.0 & 26.4 & 33.9 & \\
\hline Professional role (\%) & & & & $32.9 * * * 1$ \\
\hline Health worker & 76.0 & 47.1 & 20.0 & \\
\hline Business & 16.0 & 19.6 & 18.2 & \\
\hline Education & 4.0 & 3.9 & 7.3 & \\
\hline Other professional & 4.0 & 21.6 & 21.8 & \\
\hline Other - low income & 0 & 7.8 & 32.7 & \\
\hline
\end{tabular}

DECOA Decade of Arrival; \# Four subjects were born locally; ${ }^{* *} \mathrm{p}<0.01$, ${ }^{* * *} \mathrm{p}<0.001$; ns, not significant; ${ }^{1}$ Exact $\mathrm{p}$ - value

Participants were asked to indicate the decade closest to the year of arrival in Australia. Table 2 shows the trend of their arrival indicating the majority arrived during the period of 2000-2009 (40.6\%) and 1980-1989 (27.5\%).

Table 2. Number of Immigrant Chinese and Period of Arrival in Australia.

\begin{tabular}{ll}
\hline Period of Arrival & Number of Immigrant Chinese (Frequency, \%) \\
\hline $1950-1959$ & $4(2.9)$ \\
$1960-1969$ & $3(2.2)$ \\
$1970-1979$ & $18(13)$ \\
$1980-1989$ & $38(27.5)$ \\
$1990-1999$ & $15(10.9)$ \\
$2000-2009$ & $56(40.6)$ \\
\hline
\end{tabular}

Since the number of arrival was not even, the decade of arrival was re-specified into three categories: DECOA 1 (1950-1979), DECOA 2 (1980-1999) and DECOA 3 (20002009) attempting to have even number of participants for group comparison at a later stage. Hence, there were 25 arrivals during DECOA 1, 53 in DECOA 2, and 56 in DECOA 3. The pattern indicated that majority of immigrants arrived in 1980-2009.

\subsection{Nominated Ethnic Background}

As the ethnicity of participants was purposively selected, all participants identified as ethnic Chinese (100\%) although some of whom were born outside China, in countries like Singapore, Malaysia, Taiwan and Hong Kong.

\subsection{Highest Education Level}

As indicated in Table 1, majority of the subjects have attended undergraduate course, some of whom have postgraduate education, with a very small number only have primary or secondary school education. A Chi-square test indicated a significant association between education level and decade of arrival, $\chi^{2}(1, n=134)=22.69, p=0.007$ (Exact).

\section{Item Development of CVS - Available on Request from TKK}

The conceptual model underpinning development of the CVS was adopted from the Individualism-Collectivism Constructs pioneered by Triandis and Hofstede [1] [2] [3]. Item selections were adapted in thesis by $\mathrm{Ku}$ [7] and Hsiao [10], both are ethnic Chinese and familiar with collectivist values. Four items were dropped on the 26 items of the CVS due to their low loadings and their incoherence with any of the factors. These are item 2, 3, 16 and 21. Initially when designing the study, item 2 ('happiness unrelated to family'), item 3 ('resent invasion of privacy') and item 16 ('should live independently') were regarded as an individualist values whereas item 21 ('obligation to return favour to boss') was intended to correlate with collectivist value. Conceptualisation of these items is open for interpretation as a result of generational changes in values system, for instance in present days it is common for Chinese immigrants (students and young professionals) to live independently in Australia and that most people resent invasion of privacy.

Responses were rated on a five-point Likert scale with a score representation of ' 1 ' for 'disagree', '2' for 'tend to disagree', ' 3 ' 
for 'neither', '4' for 'tend to agree' and ' 5 ' for 'agree'.

\subsection{Procedure}

Chinese people were invited to participate and the purpose and nature of the study were explained, after ethics approval was granted by RMIT University Human Research Ethics Committee (Project No 26/09). The recruitment method was through a snow balling technique. An initial pool of Chinese $(n=20)$ was identified to be asked to participate in the study and to provide access to other participants. Chinese people who expressed an interest in participating were invited to meet with the researcher for the purpose of further explanation of the nature and procedure of the study.

\subsection{Response Rate}

Two hundred and fifty-three questionnaires were distributed either in person or via mail after initial contact with the first author. One hundred and forty-four questionnaires were returned. Six were incomplete and excluded from the analysis. So, 138 participants out of the 144 relevant respondents returned the questionnaire giving a response rate of $55 \%$.

\subsection{Statistical Analysis}

A number of preliminary Principal Component Analyses
(PCA) were conducted to explore the dimensionality of the CVS. The initial exploration revealed generally seven factors having eigenvalues greater than or equal to one. Scree plot indicated that four solutions were reasonable enough to examine further for their coherence. Prior to performing PCA, the suitability of data for factor analysis was assessed. The Kaiser-Meyer-Olkin value was 0.67 , fitted within the recommended value of 0.6 [11] [12] and Barlett's Test of Sphericity reached statistical significance $(\mathrm{p}<0.001)$ and Chisquare value of 884.87 [13], supporting the factorability of the correlation matrix. Analyses were conducted using the Statistical Package for the Social Sciences (SPSS Version 20).

\section{Results}

\subsection{Dimensions of the CVS}

A single summation of the items was calculated (after dropping the four items) to reflect appropriate interpretation of cultural values, the alpha coefficient was 0.53 , but after items 15 and 23 were reverse scored, the alpha coefficient was 0.62 indicating the 22 items yielded moderate internal consistency. The final four 'conceptual' dimensions were identified. Table 3 shows the final solution indicating the rotated component matrix, explaining $15.8 \%, 11.6 \%, 9.8 \%$ and $9.7 \%$ of the variance respectively.

Table 3. Rotated Component Matrix for the CVS Scale (Cultural Value Scale).

\begin{tabular}{|c|c|c|c|c|c|}
\hline Item & F 1 & F 2 & F 3 & F 4 & Communality \\
\hline Q 11 bring shame \& guilt & 0.72 & 0.05 & -0.04 & -0.06 & 0.50 \\
\hline Q 8 jealous of friends' achievement & 0.67 & -0.15 & 0.26 & -0.09 & 0.62 \\
\hline Q 13 eldest son have best inheritance & 0.64 & 0.07 & -0.15 & 0.18 & 0.47 \\
\hline Q 7 successful for family sake & 0.63 & 0.22 & 0.02 & -0.02 & 0.45 \\
\hline Q 5 struggle through problem & 0.58 & -0.21 & -0.04 & 0.16 & 0.40 \\
\hline Q 14 husband as breadwinner & 0.58 & 0.03 & -0.18 & -0.16 & 0.40 \\
\hline Q 19 work without pay in family business & 0.50 & 0.01 & -0.32 & 0.05 & 0.35 \\
\hline Q 17 not to do bad deed to disgrace family & 0.20 & 0.66 & 0.10 & -0.12 & 0.50 \\
\hline Q 10 parents should rely on children & 0.06 & 0.64 & -0.08 & 0.15 & 0.44 \\
\hline Q 22 family are best to rely on & 0.21 & 0.60 & 0.16 & -0.01 & 0.42 \\
\hline Q 4 live close to family & -0.15 & 0.46 & 0.06 & -0.13 & 0.25 \\
\hline Q 1 help relative with finance & -0.25 & 0.42 & -0.05 & -0.01 & 0.25 \\
\hline Q 26 student not afraid pointing out teacher's mistake & -0.15 & 0.06 & 0.76 & 0.08 & 0.61 \\
\hline Q 24 assertive to speak for own right & -0.14 & 0.19 & 0.73 & 0.09 & 0.60 \\
\hline Q 15 married only leave home \# & -0.54 & -0.13 & 0.46 & 0.01 & 0.52 \\
\hline Q 9 can only count on yourself & 0.29 & 0.14 & 0.31 & 0.46 & 0.41 \\
\hline Q 20 do what I feel best despite family & -0.07 & -0.03 & -0.08 & 0.80 & 0.66 \\
\hline Q 12 parents' opinion not important & -0.01 & -0.31 & -0.08 & 0.65 & 0.52 \\
\hline Q 25 young adults make decision without parents & 0.14 & -0.23 & 0.36 & 0.65 & 0.63 \\
\hline F 18 enjoy life to fullest & -.09 & 0.28 & -0.01 & 0.50 & 0.33 \\
\hline Percent variance & 15.8 & 11.6 & 9.8 & 9.7 & Total variance $=47.0$ \\
\hline Cronbach's alpha & & 0.77 & 0.67 & 0.54 & 0.61 \\
\hline
\end{tabular}

\# Reversed score; F 1: CFC; F 2: CFI; F 3: ISA; F 4: ISO

The first factor was labelled Collectivist Family Conformity (CFC) as the seven items relate well with collectivist values such as jealous of friends' achievement (item 8)while at the same time, struggling through a problem and aiming to be successful for family sake (item 7) and bringing shame and guilt if a family member fails badly (item
11). Although item 5 could be conceptualised as an individualistic value aiming to be independent through struggling a problem by self, in view of the literature which states that there is a high tendency for Chinese people to hide their mental illness, item 5 co-relates well with the literature and is considered as a family issue in this study. Factor two 
was labelled as Collectivist Family Interdependence (CFI). These are items relating to living close to family and providing mutual support to relatives, particularly younger generations towards the elders (senior citizen).

Factor three was labelled Individualist Self Assertion (ISA) which included item 15 and 23 (loaded negatively) supporting the assumptions that individualistic values corelate well with the notion of having intimacy in public reflecting Western culture and support the idea that youngsters do not have to leave home until they get married. These two items were reversed to obtain a positive score as shown in Table 3. The last factor was termed Individualist Self Opinion (ISO) reflecting adoption of self-interest and disregarding parents' opinions in making decision reflecting individualistic values [7] [10].

\subsection{Group Contrast of CVS Scores}

Two-way analysis of variance was conducted to examine differences between age category and decade of arrival. The first analysis was directed to examining Collectivist Family Conformity (CFC) scores. The main effect of age category was statistically significant $(\mathrm{F}(2(125)=5.12, \mathrm{p}<.01)$. Post Hoc testing using Tukey's HSD procedure $(\alpha=0.05)$ indicated significant difference for two of the three pairwise comparisons. Specifically, Age Category $3(p=0.009)$ was significantly different to Age Category $1(\mathrm{p}=0.048)$ and Age Category $2(\mathrm{p}=0.048)$. Examination of the means indicated that younger Chinese (18-34) endorsed higher level of family conformity $($ mean $=18.02$, s.d. $=5.60)$ than older Chinese $(35-$ 49) $($ mean $=18.00$, s.d.5.97) and the oldest age group $(50+)$ (mean $=14.85$, s.d. $=4.77$ ) respectively. The main effect was not statistically significant for decade of arrival $(\mathrm{F}(2(125)=1.32, \mathrm{p}>0.05)$ and neither was the interaction term $(\mathrm{F}(4(125)=0.78, \mathrm{p}>0.05)$ showing that collectivist family conformity did not vary across decade of arrival. The overall mean was 2.81 (s.d.=0.93) favouring the 'neither' response. In addition, $37.7 \%$ of the sample had an item average of greater than 3, indicating that they either 'tend to agree' or 'agree' in endorsing collectivist family conformity value.

The second analysis focused on examining collectivist family interdependence. The main effect was not statistically significant for age category $(\mathrm{F}(2,125)=0.57, \mathrm{p}>0.05)$, decade of arrival $(\mathrm{F}(2,125)=0.83, \mathrm{p}>0.05)$ or the interaction term $(\mathrm{F}(4,125)=1.24, \quad \mathrm{p}>0.05) \quad$ indicating that family interdependence did not vary across age category and decade of arrival among Chinese immigrants. The overall mean was 4.04 (s.d.=0.65) implying that collectivist family interdependence was at the 'tend to agree' response category. In addition, $90.6 \%$ of the sample had an item average of above 4 , indicating that they 'agree' to endorse the value of collectivist family interdependence.

The third analysis focused on examining Individualist Self Assertion (ISA). The main effect was not statistically significant for age category $(\mathrm{F}(2,125)=.17, \mathrm{p}>0.05)$, decade of arrival $(\mathrm{F}(2,125)=.12, \mathrm{p}>0.05)$ or the interaction term $(\mathrm{F}(4,125)=.22, \mathrm{p}>0.05)$ showing that exercising self-assertion did not vary across age category and time of arrival among
Chinese immigrants. The overall mean was 3.25 (s.d. $=0.88$ ) showing that self-assertion was at the 'neither' scale response. In addition, $60.1 \%$ of the sample had an item average of above 3 , indicating that they either 'tend to agree' or 'agree'.

The last analysis focused on examining Individualist Self Opinion (ISO). The main effect was not statistically significant for age category $(\mathrm{F}(2,125)=1.34, \mathrm{p}>0.05)$, decade of arrival $(\mathrm{F}(2,125)=.55, \mathrm{p}>0.05)$ or the interaction term $(\mathrm{F}(4,125)=1.79, \mathrm{p}>0.05)$ suggesting that exercising selfopinion did not vary across age group and time of arrival in Australia by Chinese immigrants. The overall mean was 2.88 (s.d.=0.84) suggesting that self-opinion was between the 'tend to disagree' and 'neither' but favouring the latter response category. In addition, $33.3 \%$ of the sample had an item average of greater than 3 , indicating that they either 'tend to agree' or 'agree'.

\section{Discussion}

Overall results suggest that Chinese immigrants endorsed a stronger collectivist values than individualist values which correlate positively with the literature which commented that Chinese people maintained collectivist values though living in Western nations [8] [14]. The finding indicated that participants endorsed more collectivist value $(37.7 \%$ (CFC) + $90.6 \%(\mathrm{CFI})=128.3 \%)$ than individualist value $(60.1 \%$ (ISA) $+33.3 \%$ (ISO) $=93.4 \%$ ). It is reassuring to older Chinese Australians in noting the maintenance to collectivist values, particularly with reference to Collectivist Family Conformity (CFC), given that the younger age groups (mean= 18.02 and 18.00) endorsing higher level of CFC than the oldest age group $($ mean $=14.85)$.

As the $\mathrm{PhD}$ study is based on a conceptual model incorporating the Chinese concept of face and social comparison theory, some insight into the relationship between Chinese collectivist values and stigma of mental illness using social distancing scale and negative stereotyping attitudes [7] [15] [16] could broaden this understanding. Face, in the Chinese culture, represents a Chinese person's public image [17] [18], but beyond the Western concept of the acquisition and management of others' impression of oneself, it also represents the image of one's important in-group, the family. It is an essential effort to understand the specific mechanisms in the relationship between Chinese cultural values, face concern, and social comparison. Thus, Pearson correlation was conducted to further examine variables indicating face value, social comparison and stigma.

Correlations were inspected (post hoc) between items comprising the collectivist scales (CFC and CFI) and social distancing and negative stereotyping attitudes towards mental illness. The highest correlations with social distancing were found for the three items referring to face management: 'struggle through a problem by myself' $(\mathrm{r} \quad(138)=0.31$, $\mathrm{p}<0.001$ ), 'avoid failure to avoid shame to family' ( $\mathrm{r}(138)=$ $0.37, \mathrm{p}<0.001)$ and 'husband rather than wife as breadwinner' $(\mathrm{r}(138)=0.32, \mathrm{p}<0.001)$. These respectively refer to face 
saving, traditional Chinese values and associated with social comparison. In addition, the latter two items were correlated with negative stereotyping $(\mathrm{r}(138)=0.26, \mathrm{p}<0.01)$ and $\mathrm{r}$ $(138)=0.21, \mathrm{p}<0.05)$.

In a previous study completed by $\mathrm{Ku}$ and $\mathrm{Ha}$ [14] which examined a sample of 83 Chinese nurses, items such as 'avoid bad deeds to avoid disgracing the family' ( $\mathrm{r}(83)=$ $0.32, \mathrm{p}<0.001)$ and 'achieve success for the honour of the family' $(\mathrm{r}(83)=0.43, \mathrm{p}<0.001)$ were correlated with social distancing; the latter item was correlated with negative stereotyping $(\mathrm{r}(83)=0.26, \mathrm{p}<0.05)$. It may be speculated that Chinese people hold a set of concerns for face and social comparison influencing stigma attached to mental illness. Potential stigmatisers who hold higher (or lower) concerns for face prefer to disassociate themselves from individuals who may be subject to social stigma (e.g., people with a mental illness), thus, preserving their own view of high selfstatus in society. On the other hand, if they succumbed to mental illness, they, like what had been documented in the literature [19] might avoid situations that could expose their conditions to others to avoid ridicule from others, and potentially reaffirm the precarious social status afforded to them by their mental illness.

\section{Limitation and Recommendation}

In view of the small data set obtained via convenience sampling, findings may not be representative of Chinese community's cultural values in Australia. The younger age groups (almost of equal size, 53 in DECOA 2 and 56 in DECOA 3) of immigrant Chinese had endorsed more collectivist values. Future study could replicate the same model, but should aim for a purposive sample of equal size in age category and decade of arrival, or concentrate on examining young Chinese adults, born locally in Australia to obtain a more reliable comparative result between individualistic and collectivistic values.

There is also the need to extend CVS by examining more item contents, i.e., test-retest reliability and validity indicators to capture more dimensions with other cultural groups (Europeans, Africans, Asians). The CVS can be modified to explore how saving face and social comparison may vary in individualistic nations by examining people of English descent.

\section{Conclusion}

Despite methodological limitations, the study has generated results which were previously unobtainable in Australia. Furthermore, the development of the CVS provides a measure of four key dimensions of the Individualism-Collectivism Constructs that contributes to a clearer understanding of cultural values system among Chinese immigrants in Australia. This is useful information for providing cultural responsiveness mental health services not exclusively in Australia, but also relevant in other Western nations (particularly English-speaking countries of the USA, the UK, Canada and New Zealand) where there has been an influx of Chinese immigrants in the last four decades.

\section{References}

[1] Triandis (1990). Cross-cultural studies on individualism and collectivism. In: J. J. Berman (Ed.), Nebraska Symposium on Motivation, 1989: Cross-cultural Perspectives, 37, 41-135. Lincoln, NE: University of Nebraska.

[2] Traindis (2001) - Individualism - collectivism and personality. Journal of Personality, 69(6), 907-924.

[3] Hofstede, G. H. (2001). Culture's Consequences: Comparing Values, Behaviours, Institutions, and Organisations across Nations (2nd Edition). Thousand Oaks, California: Sage.

[4] King, A. Y. \& Bond, M. H. (1985). The Confucian paradigm of man: A sociological view. In W. S. Tsang \& D. H. Wu (Eds). Chinese Culture and Mental Health, 29-45. Orlando, FL: Academic Press.

[5] Yang, K. S. (1995). Chinese Social Orientation: An Integrative Analysis. In: T-Y Lin, W-S Tsang \& E-K Yeh (Eds). Chinese Societies and Mental Health, 19-39. New York. Oxford University Press.

[6] Zhou, Y. (2008). The Modern Significance of Confucianism. Asian Social Science, 4, 11, 13-16.

[7] Ku T. K. (2007). Culture and Stigma of Mental Illness: A Comparison of General and Psychiatric Nurses of Chinese and Anglo-Australian Background. Master Thesis, Department of Psychiatry, The University of Melbourne. $\mathrm{http} / /$ repository.unimelb.edu.au/10187/8400.

[8] Fan, C. \& Karnilowicz, W. (2000). Attitudes towards Mental Illness and Knowledge of Mental Health Services among the Australian and Chinese Community. Australian Journal of Primary Health Interchange, 6, 38-48. http://dx.doi.org/10.1071/PY00017

[9] Lin T. (1983). Psychiatry and Chinese Culture. The Western Journal of Medicine, 139, 862-867.

[10] Hsiao, F. H. (2002). Chinese-Australian Families' helpseeking behaviour for mental illness. PhD Thesis. Department of Psychiatry, The University of Melbourne, Australia.

[11] Kaiser, H. (1970). A second generation Little Jiffy. Psychometrika, 35, 401-415.

[12] Kaiser, H. (1974). An index of factorial simplicity. Psychometrika, 39, 31-36.

[13] Barlett, M. S. (1954). A note on the multiplying factors for various chi square approximations. Journal of the Royal Statistical Society, 16 (Series B), 296-298.

[14] Ku, T. K. \& Ha, M. (2015). Culture and Stigma of Mental Illness: Path Analysis Conducted with AMOS in Transcultural Psychiatry in Australia. Journal of Biosciences and Medicine, $3,15-22$.

[15] http//dx.doi.org/10.4236/jbm.2015.36003.

[16] Ku, T. K. \& Ha, M. (2015). Stigma of Mental Illness: Social Distancing Attitudes among Registered Nurses in Australia. Journal of Biosciences and Medicines, 3, 40-47. 
[17] Ku T. K. \& Ha M. (2015). Negative Stereotyping Attitudes towards Mental Illness: Is it Culturally related? Journal of Biosciences and Medicine, 3, 32-39.

[18] Hu, H. C. (1944). The Chinese Concept of Face. American Anthropologist, 46, 45-64.
[19] Hwang, K. K. (1987). Face and Favour: The Chinese Power Game. American Journal of Sociology, 92, 944-974.

[20] Lee, L., Lee, M. T. Y., Chiu, M. Y. T. \& Kleinman, A. (2006). Experience of social stigma by people with schizophrenia in Hong Kong. British Journal of Psychiatry, 186, 153-157. 